\title{
A PCA Based Approach for Quantitative Analysis of Shiny Skin
}

\author{
T. Naoki, Y.W. Chen \\ Graduate School of In formation Science and Engineering, \\ Ritsumeikan University \\ Japan

\section{T. Igarashi} \\ Graduate School of In formation Science and Engineering, \\ Ritsumeikan University \\ Kao corporation, Japan
}

\begin{abstract}
We propose shiny analysis framework accompanied with makeup deterioration using normalized facial images (MaVIC and the corresponding makeup-deteriorated data sets). These images are analyzed and reconstructed based on principal component analysis (PCA) and then the differential ones between the reconstruction images with different numbers of PCA components can be generated. The shiny Eigen-faces contributing to large shiny transition can automatically be selected according to the total variances of the difference images, and then variances of the reconstructed images with only the shiny Eigen-faces can be used as measurement of shine degree. In addition, we execute PCA analysis in several color spaces, and explore the possibility of the color space, which can best manifest the shine existing in the facial images.
\end{abstract}

Keywords-facial appearance; principal component analysis (PCA); shine; MaVIC; makeup deterioration

\section{INTRODUCTION}

Most of the women do makeup in daily life, and how to enhance facial impression due to makeup types (different facial appearance) is hot-focused. Hence, quantitative assessment or analysis of facial appearance is an important issue in cosmetic foundation design [1]. Establishing these analysis methods prospects to contribute to cosmetics development. Analysis of cosmetic effect can be divided into 2 categories; variation of beautification degree between natural faces and cosmetic faces, and process (time-shift) of cos metic effect. This paper focuses on process (time-shift) of cos metic effect. Its main properties are that as the time after makeup goes away, the makeup comes off and strong shine or pigmentation irregularities appear.

In related research on analysis of makeup deterioration, Igarashi et al. proposed to use the statistics of cheek skin regions [2]. They proved that the variance and skewness of the cheek regions increase with deterioration progress of makeup. However, facial impression not only depends on the cheek regions but also be affected by global face. Moriguchi et al. proposed to access cosmetic beautification using global faces [3], which uses principal component analysis (PCA) for analysis. This study aims to analyze the shine in makeup deteriorated facial images, and automatically understands the

\author{
M. Seo \\ Graduate School of In formation Science and Engineering, \\ Ritsumeikan University \\ School of Science and Engineering \\ Kwansei Gaku in University \\ Japan
}

shiny Eigen-faces contributing to large shiny transition. The degree of shine in the makeup deteriorated facial image can be calculated using the variances of the reconstructed images with only the shiny Eigen-faces.

\section{SHINY SKIN ANALYSIS BY PCA}

\section{A. Extraction of Facial Texture Information}

In this paper, we use facial image database MaVIC (Multiangle View, Illumination and Cosmetic Facial Images Database) [4] and its corresponding makeup-deteriorated data sets. MaVIC has 267 natural and cosmetic images taken from Japanese females. And the makeup deteriorated dataset has 35 images. Images from both databases have been taken by the same device under same illu minations and viewpoints.

As we know that facial images contain shape and texture information. Since shine depend on only texture information, we firstly remove shape information by our developed morphing system AFIM (Automatic Facial Image Manipulation System) [5], which can warp each facial shape to the mean shape of some train ing face images. By excluding noses, mouths and eyes, we only focus on texture information to do analysis.

\section{B. Optimal Color Spaces for Shiny Skin Analysis}

In order to shiny transition degrees of different color information, we explore 4 color spaces: RGB, HSV, YIQ and $\mathrm{YCbCr}$ to do analysis. RGB color space is the most popular color space. By transforming RGB to HSV color space, we can separate color information into hues, saturations and values. YIQ and $\mathrm{YCbCr}$ color spaces are similar. ' $\mathrm{Y}$ ' means brightness. ' $\mathrm{T}$ ' indicates color from orange to cyan, and ' $\mathrm{Q}$ ' denotes color from green to magenta. ' $\mathrm{Cb}$ ' is the differential color information between ' $\mathrm{B}$ ' and ' $\mathrm{Y}$ ' and, ' $\mathrm{Cr}$ ' is the difference between 'R' and 'Y'. From the above color spaces, we choose 3 color components: ' $\mathrm{G}$, ' 'S' and ' $\mathrm{Cr}$ ' space. ' $\mathrm{G}$ ' is used by previous research, and the others are selected based on statistical results of large differences between facial images just after make-up, and 8 hours passing [6]. 


\section{Facial Analysis by PCA}

PCA is one of the statistical analysis methods, which can extract uncorrelated elements from input data with highdimension. Applied it to facial images, it is well known that the Eigen-faces with large Eigenvalues have global facial information and the ones with small Eigenvalues have local facial information. With PCA, we can decompose texture of any facial image to global and local information. Figure 1 shows an example of a facial image reconstruction by PCA.

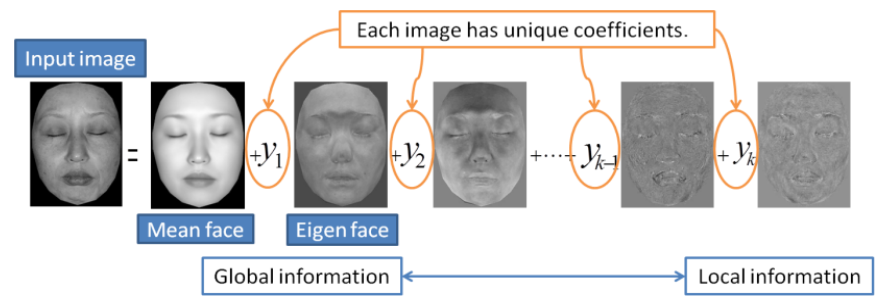

FIGURE I. AN EXAMPLE OF A FACIAL IMAGE RECONST RUCTION BY PCA.

\section{Selecting of Shiny Eigen-Faces}

We aim to find Eigen-faces contributing shiny information highly, called as shiny Eigen-faces. However, there are a lot of Eigen-faces, and then it is difficult to analy ze each one. Hence we firstly generate reconstruct images by using Eigen-faces groups with continually difference of accumulated cover rate $3 \%$, and produce the difference images in the continually reconstructed ones.

\section{E. Quantitative Assessment of Shiny Eigen-Faces}

By assuming that the Eigen-faces contribution to shiny information highly have large variance, we use the variance of the difference images for quantitative assessment. We calculate all variances of the differential images and analyze the ones with shiny Eigen-faces, which would increase with time passing. Figure 2 shows the procedure of variation calculation using difference images. Figure 3 indicates a graph of total variance. Each line indicates the variances of the differential images of the faces just after make-up, 3 hours later, 5 hours later and 8 hours later. We can find the shiny Eigen-faces contributing to shine highly from this figure and the variances of the part can be considered as quantitative measure of shine degree.

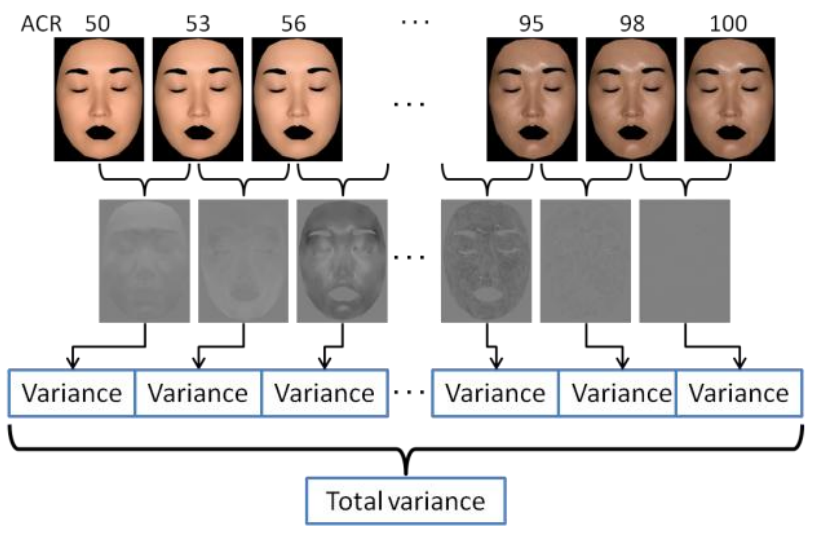

FIGURE II. A PROCEDURE OF VARIATION CALCULATION DIFFERENCE IMAGES.

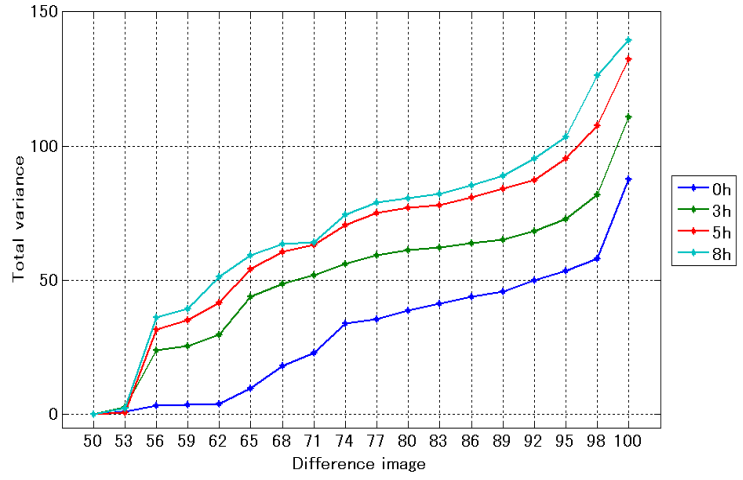

FIGURE III. A GRAPH OF TOTAL VARIANCE.

\section{EXPERIMENTS AND ANALYSIS}

We analyze typical makeup deteriorated face images in ' $G$ ', 'S' and 'Cr' color components. The results are shown in Figure 4, Figure 5 and Figure 6, respectively. It is obvious that the variances near the shiny Eigen-face increase with time passing after makeup since facial images that pass more time after make-up have more shiny information. The difference images near the shiny Eigen-faces are also shown in these Figures. The images with more passed time are enhanced in shiny regions. Comparing these 3 groups of resulted images, we can find that there are larger changes in local parts of ' $\mathrm{S}$ ' and ' $\mathrm{Cr}$ ' space than ' $G$ ' space.

The variances near the shiny Eigen-faces in all color spaces are shown in Figure 7.. We assume that the images just after makeup have no shiny information. Figure 7 shows 2 data sets results, which have similar shiny information. From Figure 7, it is obvious that the results in 'Cr' space can achieve the best performance, which correctly reflected the variability with time passing.

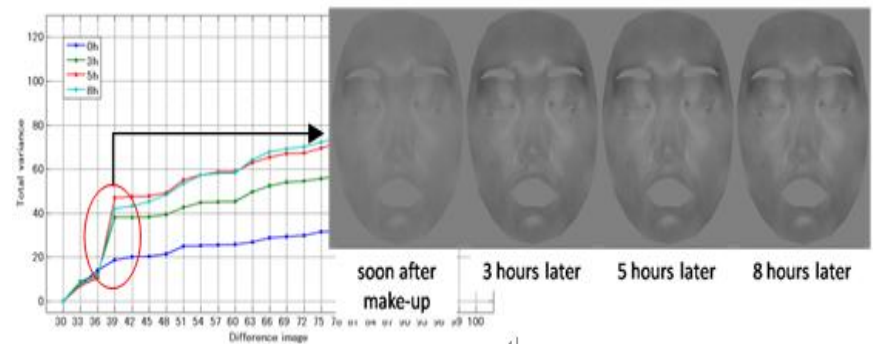

FIGURE IV. RESULT S IN G SPACE(LEFT: VARIANCE GRAPH. RIGHT: DIFFERENCE IMAGES).

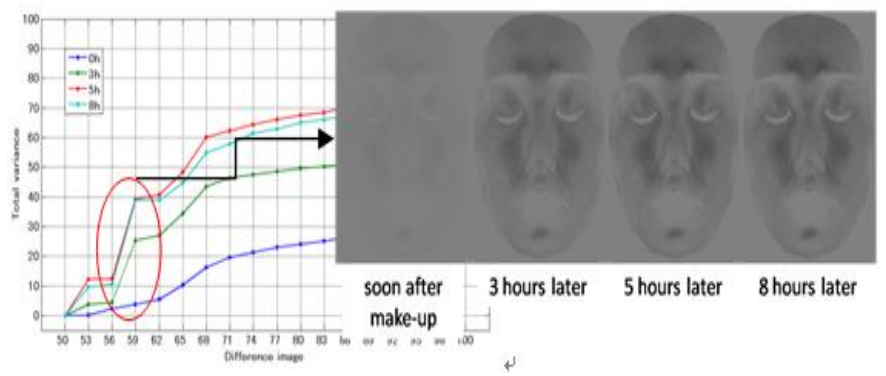

FIGURE V. RESULT S IN S SPACE(LEFT: VARIANCE GRAPH. RIGHT: DIFFERENCE IMAGES). 


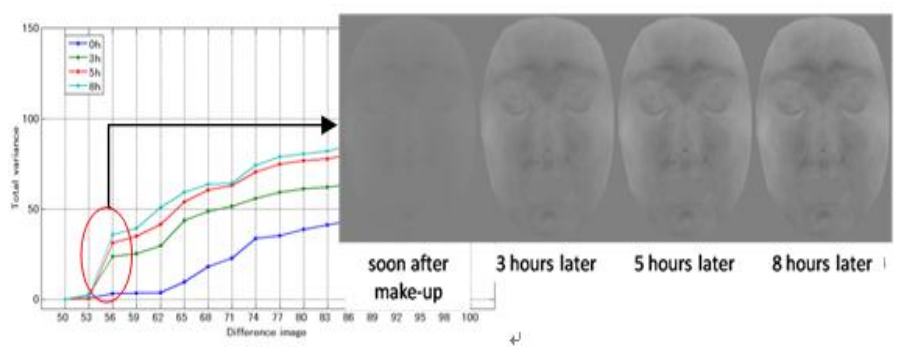

FIGURE VI. RESULT S IN CR SPACE(LEFT: VARIANCE GRAPH. RIGHT: DIFFERENCE IMAGES).
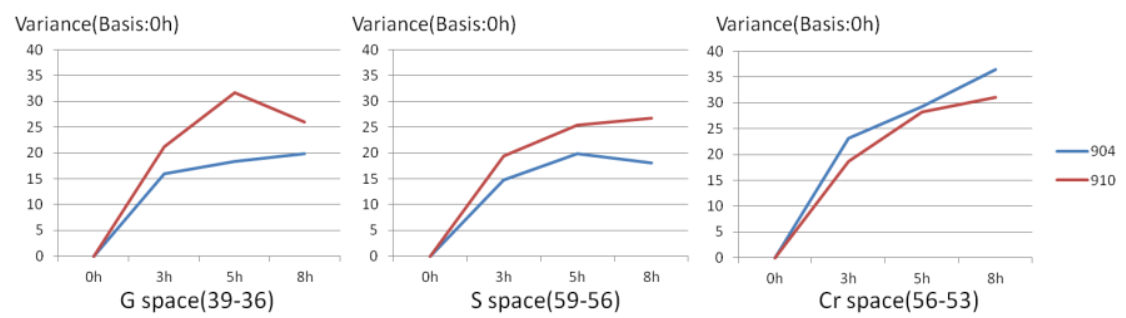

FIGURE VII.

VARIANCES OF BOUNDARY CONTRIBUTED SHINE IN EACH COLOR SPACE.

\section{CONCLUSION}

We propose a statistical analysis method for shiny information caused by makeup deterioration. We find the shiny Eigen-faces contributing to shiny information in the variance graphs that are acquired using the difference images between the reconstruction images with different numbers of PCA components. We did experiment in 3 color spaces, and validate that the ' $\mathrm{Cr}$ ' color is the optimal one for obtaining the best results.

\section{REFERENCES}

[1] T. Igarashi, "Recent technical trends on cosmetic foundations", Fragrance Journal 34, 17-28, 2006

[2] T. Igarashi et al., "Evaluation method of make-up-comes-off", Japan Patent Kokai 2009-134372, 2009

[3] J. Moriguchi et al, "Dual-Subspaces based Quantitative Analysis of Facial Appearance", Soft ware Engineering and Data Mining, pp. 652656, 2010.

[4] Y. W. Chen et al., "Multi-angle view, Illumination and Cosmetic Facial Image Database (MaVIC) and Quantitative Analysis of Facial Appearance", Lecture Notes in Computer Science, Springer, LNCS5342, pp411-420, 2008

[5] T. Terada et al.,"Automatic Facial Image Manipulation System and Facial Texture Analysis", Fifth International Conference on Natural Computation, 2009.

[6] T. Naoki et al., "Optimal Color Space for Quantitative Analysis of Shiny Skin", 6th International Conference on Biomedical Engineering and Informatics, pp.836-840, 2013. 